\title{
STABILITY OF NON-LINEAR SYSTEMS WITH TIME-VARYING DELAYS
}

\author{
R. M. LEWIS
}

(Received 18 April 1979)

(Revised 10 September 1979)

\begin{abstract}
A condition guaranteeing the stability of linear systems with time delays in the interactions among elements is generalized to cover non-linear systems and discontinuous, unbounded delays.
\end{abstract}

\section{Introduction}

In a recent paper [1], it was shown that a linear time-delayed system with bounded coefficients is stable if its system matrix is column diagonally dominant. Subsequently we proved [5] that, if the linearization of a non-linear time-delayed system satisfies the same condition, then the non-linear system is stable. The derivation in [5] is for the case when the time delays are constant and does not readily generalize-it is particularly difficult to establish the boundedness of the solutions when the delays are time varying (see [5], appendix 3).

Here we obtain stability results for the time-varying delay case by strengthening the diagonal dominance condition; it should, however, be noted that for linear systems the new and old conditions are identical. The stronger condition permits more direct proofs than those given in [5]. The restrictions on the time delay functions are quite weak; in fact, unbounded delays can be dealt with.

We noted in [5] that the column diagonal dominance condition used there could be replaced by one of quasi-diagonal dominance and the same applies here. We refer to [1], [4] and [5] and the references contained therein for wider discussions on the stability of time-delayed systems. 


\section{System description}

The systems considered are the same as in [5] but with time-varying delays (and no inputs). They are described by the following $n$th order homogeneous differential delay equations on the half line $[0, \infty)$ :

$$
\dot{x}_{i}(t)=-f_{i 0}\left(x_{i}(t)\right)+\sum_{j \neq i} f_{i j}\left(x_{j}\left(t-T_{i j}(t)\right)\right), \quad i=1, \ldots, n .
$$

(Here $\sum_{j \neq i}$ is an abbreviation of $\sum_{\substack{j=i \\ j \neq i}}^{n}$ ) The vector $x(t)=\left[x_{1}(t), \ldots, x_{n}(t)\right]^{\mathrm{T}}$ is called the instantaneous system state and the function $x(\cdot):[0, \infty) \rightarrow \mathbf{R}^{n}$, the system trajectory. The complete system state

$$
x_{\imath} \Delta\{x(s): t-T(t) \leqslant s \leqslant t\}, \quad \text { where } T(t)=\max _{i, j} T_{i j}(t),
$$

is infinite dimensional. System (2.1) is autonomous in the sense that

$$
f_{i 0}, f_{i j}, \quad i, j=1, \ldots, n,
$$

are time independent. Non-autonomous systems are not considered here; some results for the linear case with constant delays are given in [5].

The scalar functions $T_{i j}(\cdot)$ are called time delays and are required to satisfy, for all $i, j=1, \ldots, n, i \neq j$ :

\section{ASSUMPTION 2.1}

(a) $T_{i j}(\cdot)$ is a function of bounded variation and is right continuous on each bounded interval $[0, \tau] \subset[0, \infty)$,

(b) $T_{i j}(t) \geqslant 0$ for all $t \in[0, \infty)$,

(c) $t-T_{i j}(t)$ is monotone non-decreasing.

REMARKs. (i) (a) implies that $T(t)=\max _{i, j} T_{i j}(t)<\infty$.

(ii) (b) is necessary if (2.1) is to represent a causal system.

(iii) (c) is the natural requirement that, if $s \geqslant t, \dot{x}(s)$ depends upon later values of $x(\cdot)$ than does $\dot{x}(t)$.

The solution of (2.1) depends upon the specification of an initial condition, $x(s)=\phi(s), s \in[-T(0), 0]$. We shall assume that the given $n$ vector function $\phi(\cdot)$ is absolutely continuous and for simplicity take each component $x_{i}(\cdot)$ to be specified on the same initial interval.

To discuss stability we must be sure that the solution to (2.1) exists on $[0, \infty)$ and therefore assume that the functions $f_{i 0}, f_{i j}, i, j=1, \ldots, n$, are such that corresponding to any initial condition (2.1) has a unique absolutely continuous solution on $[0, \infty)$. In particular we assume that $f_{i 0}, f_{i j}$ are continuously 
differentiable, hence locally Lipschitz. Systems which admit trajectories with finite escape times or trajectories which fail to exist beyond a certain point are excluded (see [4]).

The results in this paper deal with the asymptotic stability of the equilibrium point of (2.1). Without ambiguity we henceforth refer to this as stability. The conditions imposed upon the functions $f_{i 0}, f_{i j}$ will ensure that a unique equilibrium point exists. Our approach involves the second derivative system obtained from (2.1) but, because we admit delays with discontinuities, the second derivatives are not absolutely continuous and do not satisfy a differential equation. Let $z(t)=\dot{x}(t) ;$ then we can write:

$$
\begin{array}{r}
d z_{i}(t)=-a_{i 0}(t) z_{i}(t) d t+\sum_{j \neq i} a_{i j}\left(t-T_{i j}(t)\right) z_{j}\left(t-T_{i j}(t)\right]\left[d t-d T_{i j}(t)\right], \\
i=1, \ldots, n,
\end{array}
$$

where $d T_{i j}\left({ }^{\cdot}\right)$ is a Borel measure on $[0, \tau]$ for all $\tau<\infty$, corresponding to the function $T_{i j}(\cdot)$ of bounded variation. The coefficients $a_{i 0}(\cdot)$ and $a_{i j}(\cdot)$ are defined by

and

$$
a_{i 0}(t)=d f_{i 0}(\sigma) / d \sigma, \text { where } \sigma=x_{i}(t)
$$

$$
a_{i j}(t)=d f_{i j}(\sigma) / d \sigma, \text { where } \sigma=x_{j}(t) .
$$

Let $\mu_{i j}(t)=t-T_{i j}(t), i, j=1, \ldots, n, i \neq j$; then (2.2) can be regarded as a formal version of the integral equation

$$
\begin{array}{r}
z_{i}(t)=z_{i}(0)+\int_{0}^{t}-a_{i 0}(s) z_{i}(s) d s+\sum_{j \neq i} \int_{0}^{t} a_{i j}\left(\mu_{i j}(s)\right) z_{j}\left(\mu_{i j}(s)\right) d \mu_{i j}(s), \\
\quad i=1, \ldots, n .
\end{array}
$$

In view of the fact that the measures $\mu_{i j}(\cdot)$ correspond to functions of bounded variation, the solutions to (2.3) satisfy (2.2) almost everywhere with respect to $\mu=t+\sum_{i=1}^{n} \sum_{j \neq i} \mu_{i j}(t)$ and the properties of $z(\cdot)$ can be determined from either (2.2) or (2.3) (see [3, Theorem 1]). We refer to both as the integral equation for $z(\cdot)$. In the following section the stability of solutions to the integral equation is analysed.

\section{Stability of a linear integral equation}

Here the methods introduced in [1] are extended to the integral equation (2.2). First we define a positive system whose trajectories dominate those of (2.2) and then, using a linear Lyapunov-like function, we show that the dominating system is stable, under suitable conditions. 
LEMMA 1. If $z(t)$ solves (2.2) then $|z(t)|=\left[\left|z_{1}(t)\right|, \ldots,\left|z_{n}(t)\right|\right]^{\mathrm{T}}$ satisfies

$$
\begin{aligned}
& d\left|z_{i}(t)\right| \leqslant-a_{i 0}(t)\left|z_{i}(t)\right| d t+\sum_{j \neq i}\left|a_{i j}\left(\mu_{i j}(t)\right)\right|\left|z_{j}\left(\mu_{i j}(t)\right)\right| d \mu_{i j}(t), \\
& i=1, \ldots, n,
\end{aligned}
$$

almost everywhere with respect to $\mu$ defined above.

Proof. As each $\mu_{i j}(\cdot)$ is right continuous (Assumption 2.1(a)), each $z_{i}(\cdot)$ and hence $\left|z_{i}(\cdot)\right|$ is right continuous (see $\left.[2,3]\right)$. Consequently $d z_{i}(t)=z_{i}(t)-z_{i}\left(t^{-}\right)$ and $d\left|z_{i}(t)\right|=\left|z_{i}(t)\right|-\left|z_{i}\left(t^{-}\right)\right|$.

There are two cases to consider:

(i) $z_{i}(t) \geqslant 0$.

$$
\begin{aligned}
d\left|z_{i}(t)\right| & =z_{i}(t)-\left|z_{i}\left(t^{-}\right)\right| \\
& \leqslant d z_{i}(t) \\
& =-a_{i 0}(t) z_{i}(t) d t+\sum_{j \neq i} a_{i j}\left(\mu_{i j}(t)\right) z_{j}\left(\mu_{i j}(t)\right) d \mu_{i j}(t) \\
& \leqslant-a_{i 0}(t)\left|z_{i}(t)\right| d t+\sum_{j \neq i}\left|a_{i j}\left(\mu_{i j}(t)\right)\right|\left|z_{j}\left(\mu_{i j}(t)\right)\right| d \mu_{i j}(t)
\end{aligned}
$$

since, by Assumption $2.1(\mathrm{c}), d \mu_{i j}(t) \geqslant 0$ for all $i, j$.

(ii) $z_{i}(t)<0$.

$$
\begin{aligned}
& d\left|z_{i}(t)\right|=-z_{i}(t)-\left|z_{i}\left(t^{-}\right)\right| \\
& =-d z_{i}(t)-z_{i}\left(t^{-}\right)-\left|z_{i}\left(t^{-}\right)\right| \\
& \leqslant-d z_{i}(t) \\
& =a_{i 0}(t) z_{i}(t) d t-\sum_{j \neq i} a_{i j}\left(\mu_{i j}(t)\right) z_{j}\left(\mu_{i j}(t)\right) d \mu_{i j}(t) \\
& \leqslant-a_{i 0}(t)\left|z_{i}(t)\right| d t+\sum\left|a_{i j}\left(\mu_{i j}(t)\right)\right|\left|z_{j}\left(\mu_{i j}(t)\right)\right| d \mu_{i j}(t) .
\end{aligned}
$$

Let $y(t)$ solve

$$
d y_{i}(t)=-a_{i 0}(t) y_{i}(t) d t+\underset{j \neq i}{+} \sum\left|a_{i j}\left(\mu_{i j}(t)\right)\right| y_{j}\left(\mu_{i j}(t)\right) d \mu_{i j}(t), \quad i=1, \ldots, n,
$$

with $y(s)=|z(s)|, s \in[-T(0), 0]$.

Note. As (3.2) is linear it has a solution on $[0, \infty)$ (see [6]). The initial function $y(s)=|z(s)|=|\dot{\phi}(s)|$ is measurable.

LEMMA 2. If $z(t)$ and $y(t)$ solve (2.2) and (3.2) respectively, then $|z(t)| \leqslant y(t)$ for all $t \in[0, \infty)$. (The inequality $z \leqslant y$ denotes $z_{i} \leqslant y_{i}, i=1, \ldots, n$.)

Proof. As in [1], Lemma 2, we replace (3.2) with

$$
\begin{array}{r}
d y_{i}^{m}(t)=-a_{i 0}(t) y_{i}^{m}(t) d t+\sum_{j \neq i}\left|a_{i j}\left(\mu_{i j}(t)\right)\right| y_{j}^{m}\left(\mu_{i j}(t)\right) d \mu_{i j}(t)+m^{-1} d t, \\
i=1, \ldots, n,
\end{array}
$$

with $y_{m}(s)=y(s)$ on $[-T(0), 0]$, where $m=1,2, \ldots$ 
Suppose $\left|z_{i}(t)\right|>y_{i}^{m}(t)$ for some $t, i$; then, because of the right continuity of $z(\cdot)$ and $y_{m}(\cdot)$, there exists a minimal $T$ such that $\left|z_{j}(s)\right| \leqslant y_{j}^{m}(s)$ for all $j$ and $s \leqslant T$ and, for some $i$, for all $\varepsilon>0$ sufficiently small, $\left|z_{i}(T+\varepsilon)\right|>y_{i}^{m}(T+\varepsilon)$. The continuity properties of $z_{i}$ and $y_{i}^{m}$ imply that $\left|z_{i}(T)\right|=y_{i}^{m}(T)$, hence

$$
\begin{aligned}
d\left[\left|z_{i}(T)\right|-y_{i}^{m}(T)\right] & \leqslant-a_{i 0}(t)\left[\left|z_{i}(T)\right|-y_{i}^{m}(T)\right] d t-m^{-1} d t \\
& =-m^{-1} d t,
\end{aligned}
$$

which implies that $\left|z_{i}(t)\right| \leqslant y_{i}^{m}(t)$ to the right of $T$, a contradiction.

The proof is completed by showing that $y_{m} \rightarrow y$ pointwise on every bounded interval, as $m \rightarrow \infty$. Let $y^{a}$ denote the solution of (3.3) with $a$ replacing $m^{-1}$. The result of Warga [6, Theorem II.6.9] proves that the map $a \rightarrow y^{a}: \mathbf{R} \rightarrow L^{1}\left[I, \mathbf{R}^{n}\right]$ is continuous, for any compact interval $I \subset[0, \infty)$. Thus, as $m \rightarrow \infty, y^{m} \rightarrow y$ in $L^{1}\left[I, \mathbf{R}^{n}\right]$ and, since the functions are right continuous, $y^{m} \rightarrow y$ pointwise in $I$. We conclude that $y^{m}(t) \rightarrow y(t)$ for all $t<\infty$, hence $|z(t)| \leqslant y(t)$ as claimed.

NoTE. To apply [6, Theorem II.6.9] we have to write (3.3) in the form

This is done by writing

$$
d y_{i}^{m}=f_{i}\left(t, y_{t}^{m}, m\right) d \mu(t) .
$$

$$
\mu(t)=t+\sum_{i=1}^{n} \sum_{j \neq i} \mu_{i j}(t)
$$

defining $\eta_{i j}(t)$ and $\eta(t)$ to be the Radon-Nikodym derivatives of $\mu_{i j}(t)$ and $t$ respectively with respect to $\mu$, and setting

$$
f_{i}\left(t, y_{t}^{m}, m\right)=-a_{i 0}(t) \eta(t) y_{i}(t)+\sum_{j \neq i}\left|a_{i j}\left(\mu_{i j}(t)\right)\right| y_{j}\left(\mu_{i j}(t)\right) \eta_{i j}(t)+m^{-1} \eta(t)
$$

COROLlaRY 1. Stability of (3.2) implies stability of (2.2).

CoROLlary 2. The solutions $y(t)$ to (3.2) are non-negative, that is, $y_{i}(t) \geqslant 0$ for all $t, i=1, \ldots, n$.

Let us now consider the stability of the solutions $y(t)$ of (3.2). It is sufficient to show that $\alpha(t) y(t)$ is bounded, for some scalar function $\alpha(t)$ such that

$$
\lim _{t \rightarrow \infty} \alpha(t)=\infty
$$

If $\alpha(\cdot)$ is differentiable and positive then $r(t)=\alpha(t) y(t)$ is non-negative and

$$
d r_{i}(t)=-b_{i 0}(t) r_{i}(t) d t+\sum_{j \neq i} b_{i j}\left(\mu_{i j}(t)\right) r_{j}\left(\mu_{i j}(t)\right) d \mu_{i j}(t), \quad i=1, \ldots, n,
$$

for $\mu$ almost all $t$, where

and

$$
\left.\begin{array}{l}
b_{i 0}(t)=a_{i 0}(t)-\dot{\alpha}(t) / \alpha(t), \quad i=1, \ldots, n \\
b_{i j}(t)=\left|a_{i j}(t)\right| \alpha\left(t+T_{i j}(t)\right) / \alpha(t), \quad i, j=1, \ldots, n .
\end{array}\right\}
$$


Define

$$
V(t)=\sum_{i=1}^{n}\left\{r_{i}(t)+\sum_{j \neq i} \int_{\mu_{l,}(t)}^{t} b_{i j}(s) r_{j}(s) d s\right\} .
$$

Then $V(t)$ is of bounded variation on bounded intervals and, since each term in (3.6) is non-negative, $V(t) \geqslant 0$ for all $t$.

For $\mu$ almost all $t$,

$$
\begin{aligned}
d V(t)= & \sum_{i=1}^{n}\left\{-b_{i 0}(t) r_{i}(t) d t+\right. \\
& +\sum_{j \neq i} b_{i j}\left(\mu_{i j}(t)\right) r_{j}\left(\mu_{i j}(t)\right) d \mu_{i j}(t) \\
& \left.+\sum_{j \neq i}\left[b_{i j}(t) r_{j}(t) d t-b_{i j}\left(\mu_{i j}(t)\right) r_{j}\left(\mu_{i j}(t)\right) d \mu_{i j}(t)\right]\right\} \\
= & \sum_{i=1}^{n}\left\{-b_{i 0}(t)+\sum_{j \neq i} b_{j i}(t)\right\} r_{i}(t) d t .
\end{aligned}
$$

As $t$ is absolutely continuous with respect to $\mu,(3.7)$ implies that $V(\cdot)$ is absolutely continuous and, for almost all $t$,

$$
\dot{V}(t)=\sum_{i=1}^{n}\left\{-b_{i 0}(t)+\sum_{j \neq i} b_{j i}(t)\right\} r_{i}(t)
$$

Consequently, if, for all $t, i=1, \ldots, n$,

$$
b_{i 0}(t)-\sum_{j \neq i} b_{j i}(t) \geqslant 0,
$$

then $\dot{V}(t) \leqslant 0, V(t)$ is bounded above by $V(0)$, and $r(t)$ is bounded, as required.

Our main results are based upon showing that, subject to conditions stated below, (3.8) is verified for a suitable choice of $\alpha(\cdot)$.

THEOREM 1. If the coefficients of the integral equation (2.2) satisfy the strengthened column dominance condition:

$$
\left.\begin{array}{l}
\text { there exist } 0<\varepsilon<1 \text { and } \delta>0 \text { such that, for all } t, \\
\varepsilon a_{i 0}(t)-\sum_{j \neq i}\left|a_{j i}(t)\right|-\delta>0, \quad i=1, \ldots, n,
\end{array}\right\}
$$

and if all the time delays are bounded, then (2.2) is exponentially stable.

Proof. In terms of the coefficients of (2.2), (3.8) is

$$
a_{i 0}(t)-\dot{\alpha}(t) / \alpha(t)-\sum_{j \neq i}\left|a_{j i}(t)\right| \alpha\left(t+T_{j i}(t)\right) / \alpha(t) \geqslant 0, \quad i=1, \ldots, n .
$$

Let $\alpha(t)=\exp (k t)$ for some $k>0$ to be determined and let $T$ be an upper bound 
for all the time delays $T_{i j}(t)$. Then, subject to (3.9),

$$
\begin{aligned}
a_{i 0}(t) & -\dot{\alpha}(t) / \alpha(t)-\sum_{j \neq i}\left|a_{j i}(t)\right| \alpha\left(t+T_{j i}(t)\right) / \alpha(t) \\
& \geqslant a_{i 0}(t)-k-\exp (k T)\left[\varepsilon a_{i 0}(t)-\delta\right] \\
& =a_{i 0}(t)[1-\varepsilon \exp (k T)]-k+\delta \exp (k T) \\
& \geqslant 0 \text { for all } i=1, \ldots, n,
\end{aligned}
$$

provided $k \leqslant(1 / T) \log (1 / \varepsilon)$ and $k \leqslant \delta \exp (k T)$. These inequalities are satisfied by $k=\min [(1 / T) \log (1 / \varepsilon), \delta]>0$.

Remarks. (i) (3.9) implies that $a_{i 0}(t)>0$ for all $i$ and $t$.

(ii) The standard column dominance condition is expressed as

$$
a_{i 0}(t)-\sum_{j \neq i}\left|a_{j i}(t)\right|-\delta>0, \quad i=1, \ldots, n ;
$$

that is, the $\varepsilon$ factor is absent. The above proof fails if this is used in place of (3.9), unless the coefficients are $a$ priori bounded, in which case the two conditions are equivalent.

(iii) If some of the time delays are unbounded, we cannot construct an exponential $\alpha(\cdot)$ as in the above proof. Instead we have polynomial stability as indicated in the following theorem.

THEOREM 2. If the coefficients of the integral equation (2.2) satisfy the strengthened column dominance condition (3.9) and if the time delays $T_{i j}(t)$ are bounded above by $\eta t+k$, then the solutions of (2.2) go asymptotically to zero at least as fast as $t^{-p}$ provided $(1+\eta)^{p}<\varepsilon^{-1}$, for some $p>0$.

Proof. Let $\alpha(t)=t^{p}, p>0$; then with the above assumptions the left hand side of (3.10) can be written as

$$
\begin{aligned}
a_{i 0}(t) & -\dot{\alpha}(t) / \alpha(t)-\sum_{j \neq i}\left|a_{j i}(t)\right| \alpha\left(t+T_{j i}(t)\right) / \alpha(t) \\
& \geqslant a_{i 0}(t)-\frac{p}{t}-\sum_{j \neq i}\left(1+\eta+\frac{k}{t}\right)^{p}\left|a_{j i}(t)\right| \\
& \geqslant a_{i 0}(t)\left[1-\left(1+\eta+\frac{k}{t}\right)^{p} \varepsilon\right]-\frac{p}{t}+(1+\eta)^{p} \delta \\
& \geqslant 0, \quad i=1, \ldots, n,
\end{aligned}
$$

for large $t$ provided $(1+\eta)^{p}<\varepsilon^{-1}$.

Remarks. (i) Since we require $t-T_{i j}(t)$ to be monotone non-decreasing, that is, $d T_{i j}(t) \leqslant d t$, we have $T_{i j}(t)=T_{i j}(0)+\int_{0}^{t} d T_{i j}(s) \leqslant t+T_{i j}(0)$ and so, with 
the range $0 \leqslant \eta \leqslant 1$, Theorem 2 covers the entire range of admissible delays.

(ii) For any $\eta \in[0,1]$, taking $p>0$ sufficiently small we can ensure that $(1+\eta)^{p} \leqslant \varepsilon^{-1}$ (as $\left.\varepsilon<1\right)$. In the following section we are interested only in $p>1$ : given $\varepsilon$, this restricts the range of $\eta$ to $\eta<\varepsilon^{-1}-1$; given $\eta, \varepsilon$ is restricted to $\varepsilon \leqslant(1+\eta)^{-p}<(1+\eta)^{-1}$.

\section{Stability of non-linear systems}

Recalling that the solutions $x(\cdot)$ of $(2.1)$ and $z(\cdot)$ of $(2.2)$ are related by $\dot{x}(t)=z(t)$ almost everywhere in $[0, \infty)$, we use Theorems 1 and 2 to prove stability of (2.1). The coefficients $a_{i 0}(t)$ and $a_{j i}(t)$ of (2.2) are defined by

$$
a_{i 0}(t)=d f_{i 0}\left(x_{i}(t)\right) / d \sigma \quad \text { and } \quad a_{j i}(t)=d f_{j i}\left(x_{i}(t)\right) / d \sigma ;
$$

hence (3.9) is implied by

$$
\left.\begin{array}{l}
\text { there exist } 0<\varepsilon<1 \text { and } \delta>0 \text { such that, for all } \sigma \in \mathbf{R}, \\
\varepsilon d f_{i 0}(\sigma) / d \sigma-\sum_{j \neq i}\left|d f_{j i}(\sigma) / d \sigma\right|-\delta>0, \quad i=1, \ldots, n .
\end{array}\right\}
$$

THEOREM 3. If the functions $f_{i 0}(\cdot)$ and $f_{i j}(\cdot)$ defining the non-linear time-delayed system (2.1) satisfy the strengthened dominance condition (4.1) for some $\varepsilon, \delta$, and either

(a) the time delays are bounded, or

(b) the delays satisfy $T_{i j}(t) \leqslant \eta t+k$ and $(1+\eta)^{p} \leqslant \varepsilon^{-1}$ for some $p>1$, then every trajectory of (2.1) converges asymptotically to an equilibrium point of (2.1). Moreover, if (a) holds or (b) holds with $\eta<1$, the equilibrium point is unique.

Proof. (i) Stability. We have $x(t)=x(0)+\int_{0}^{t} z(s) d s$. Now (4.1) and (a) yield, from Theorem 1 , that $\|z(t)\| \leqslant m \exp (-k t)$ for some $m, k>0$. Hence $z(\cdot)$ is integrable on $[0, \infty)$ and

$$
\lim _{t \rightarrow \infty} x(t)=x(0)+\int_{0}^{\infty} z(s) d s=x_{\infty} \text { exists. }
$$

Similarly, (4.1) and (b) yield, from Theorem 2 , that $\|z(t)\| \leqslant m t^{-p}$ for some $m>0, p>1$. Therefore $z(\cdot)$ is integrable on $[0, \infty)$ and

$$
\lim _{t \rightarrow \infty} x(t)=x(0)+\int_{0}^{\infty} z(s) d s=x_{\infty} \quad \text { exists. }
$$

Clearly $x_{\infty}$ is an equilibrium point of (2.1). 
(ii) Uniqueness of the equilibrium point. When (a) or (b) with $\eta<1$ holds, the set of equilibrium points of (2.1) is given by $\{x: F(x)=0\}$, where $F: \mathbf{R}^{n} \rightarrow \mathbf{R}^{n}$ is given by $F_{i}(x)=-f_{i 0}\left(x_{i}\right)+\sum_{j \neq i} f_{i j}\left(x_{j}\right)$. Satisfaction of (4.1) implies satisfaction of the standard dominance condition with $\varepsilon=1$. In [5, Theorem 9] it is shown that this implies that the equilibrium set comprises a single point.

Notes. (i) The ease with which this result is obtained compared with the similar one [5, Theorem 3] is noteworthy. The strengthening of the dominance condition through the incorporation of the factor $\varepsilon$ enables us to prove stability directly, without first hypothesizing trajectory boundedness. In [5] boundedness was proved to be equivalent to the existence of an equilibrium point, which was then demonstrated.

A further advantage of the present result is that a wide class of time-varying delays is admitted.

(ii) When $\eta=1$ in hypothesis (b) of the theorem, delays $T_{i j}(t)=t$ are admitted. Hence $f_{i j}\left(x_{j}\left(t-T_{i j}(t)\right)\right)=f_{i j}\left(x_{j}(0)\right)$ and the equilibrium set is not given by $\{x: F(x)=0\}$. An illustrative example is given in the following section.

(iii) As mentioned in Section 1, a quasi-diagonal dominance condition is also sufficient for the stability of (2.1). Suppose we replace $V(t)$ in (3.6) by

$$
\bar{V}(t)=\sum_{i=1}^{n} d_{i}\left\{r_{i}(t)+\sum_{j \neq i} \int_{\mu_{i j}(t)}^{t} b_{i j}(s) r_{j}(s) d s\right\}
$$

for some collection of positive scalars $d_{i}$. Then

$$
\nabla(t)=\sum_{i=1}^{n}\left\{-d_{i} b_{i 0}(t)+\sum_{j \neq i} d_{j} b_{j i}(t)\right\} r_{i}(t)
$$

and stability is ensured by

$$
d_{i} b_{i 0}(t)-\sum_{j \neq i} d_{j} b_{j i}(t) \geqslant 0
$$

In the bounded delay case this is true with exponential $\alpha(\cdot)$ provided the strengthened quasi-diagonal dominance condition holds: there exist $\varepsilon \in(0,1)$ and $\delta>0$ such that, for all $i$ and $\sigma \in \mathbf{R}$,

$$
\varepsilon d_{i} d f_{i 0}(\sigma) / d \sigma-\sum_{j \neq i}\left|d_{j} d f_{j i}(\sigma) / d \sigma\right|-\delta>0 .
$$

\section{Examples}

EXAMPLE 1.

$$
\begin{aligned}
& \dot{x}_{1}(t)=-x_{1}(t) / 2-x_{1}^{3}(t) / 3+x_{2}(t-1)+1, \\
& \dot{x}_{2}(t)=-2 x_{2}(t)+x_{1}^{2}(t-2)-4 / 3 .
\end{aligned}
$$


This system is neither column nor row diagonally dominant. However, taking $d_{1}=1, d_{2}=3 / 5$ and $\varepsilon=9 / 10$,

$$
\varepsilon d_{1} d f_{10}(\sigma) / d \sigma-d_{2} d f_{21}(\sigma) / d \sigma=9 / 10\left[\frac{1}{2}+\sigma^{2}\right]-6 \sigma / 5 .
$$

This has a minimum value of $1 / 20$ at $\sigma=2 / 3$.

$$
\varepsilon d_{2} d f_{20}(\sigma) / d \sigma-d_{1} d f_{12}(\sigma) / d \sigma=27 / 50[2]-1=2 / 25 .
$$

Therefore (4.2) is satisfied by choosing $0<\delta<1 / 20$, and all the trajectories of the system converge asymptotically to the equilibrium point $\left(x_{1}, x_{2}\right)=(1,-1 / 6)$.

\section{EXAMPLE 2.}

$$
\begin{aligned}
& \dot{x}_{1}(t)=-x_{1}(t)+x_{2}(t / 2) / 2, \\
& \dot{x}_{2}(t)=-x_{2}(t)+x_{1}(t / 2) / 2 .
\end{aligned}
$$

The delays are $T_{12}(t)=T_{21}(t)=t / 2$, that is, $\eta=\frac{1}{2}$ in Theorem 3. For $\frac{1}{2}<\varepsilon<1$, (4.1) holds and so, with $1<p<\log _{3 / 2} 2$, condition (b) of Theorem 3 can be satisfied. The trajectories converge asymptotically to the equilibrium point $(0,0)$.

The minimum rate of convergence is as

$$
t^{-(p-1)}, \quad \text { where } p-1=\log _{3 / 2} 2-1=0.5874 \ldots
$$

It can be shown that the maximum rate of convergence is as $t^{-1}$.

Suppose $x_{1}(0)=x_{2}(0)>0$; then $x_{1}(t)=x_{2}(t)>0$ for all $t \in[0, \infty)$. (It is easily verified that, for such initial conditions, $x_{1}\left(t_{0}\right)=0$ for $t_{0}<\infty$ is impossible.) Let $y(t)=x_{1}\left(e^{t}\right)$; then $\dot{y}(t)=e^{t}\left(-y(t)+\frac{1}{2} y(t-\log 2)\right)$. Let

$$
V(t)=y(t)+\int_{t-\log 2}^{t} e^{s} y(s) d s>0 \text { for all } t
$$

Then

$$
\dot{V}(t)=e^{t}\left(-y(t)+\frac{1}{2} y(t-\log 2)\right)+e^{t} y(t)-\frac{1}{2} e^{t} y(t-\log 2)=0,
$$

that is,

$$
V(t)=k>0 \text { for all } t
$$

As $y(t) \rightarrow 0$ for large $t$,

$$
\begin{aligned}
k / 2 & \leqslant \int_{t-\log 2}^{t} e^{s} y(s) d s \\
& \leqslant \log 2 . e^{t} \max _{s \in[t-\log 2, t]}\{y(s)\} .
\end{aligned}
$$

Thus $y(t)$ goes to zero at most as fast as $e^{-t}$ and $x_{1}(t)=x_{2}(t)=y(\log t)$ at most as fast as $t^{-1}$. This shows that the estimate $t^{-(p-1)}$ is not overly conservative. (Note: $\log$ above denotes $\log _{e}$.) 
EXAMPLE 3.

$$
\begin{aligned}
& \dot{x}_{1}(t)=-x_{1}(t)+b x_{2}(0), \\
& \dot{x}_{2}(t)=-x_{2}(t)+b x_{1}(0), \quad b \geqslant 0 .
\end{aligned}
$$

Here, $T_{12}(t)=T_{21}(t)=t$, that is, $\eta=1$ and (4.1) holds for any $1-b<\varepsilon<1$. To guarantee convergence to an equilibrium point using Theorem 3 we must have, for some $p>1,(i-b)^{-1} \geqslant 2^{p}>2$, that is, $b<\frac{1}{2}$. However,

$$
\begin{aligned}
& x_{1}(t)=\exp (-t) x_{1}(0)+(1-\exp (-t)) b x_{2}(0) \rightarrow b x_{2}(0), \\
& x_{2}(t)=\exp (-t) x_{2}(0)+(1-\exp (-t)) b x_{1}(0) \rightarrow b x_{1}(0) .
\end{aligned}
$$

The system trajectories converge for all values of $b$; we conclude that the conditions of Theorem 3 are far from necessary. Note that the equilibrium point depends upon the initial condition.

EXAmPLE 4. Sampled-data interactions. Consider the system defined by

$$
\left.\begin{array}{rl}
\dot{x}_{i}(t) & =-a_{i 0} x_{i}(t)+\sum_{j \neq i} a_{i j} x_{j}(m T), \quad t \in[m T,(m+1) T), \\
m & =1,2, \ldots \quad \text { and } \quad i=1, \ldots, n .
\end{array}\right\}
$$

This can be written in our standard form by taking

$$
T_{i j}(t)=t-m T, \quad t \in[m T,(m+1) T) \text { for all } i, j=1, \ldots, n, \quad i \neq j .
$$

Then $T_{i j}(t) \leqslant T$ and if the coefficients $a_{i} 0$ and $a_{i j}$ satisfy the strengthened diagonal dominance condition, the trajectories of (5.1) converge exponentially to zero.

Note that the delays in the example are of bounded variation on bounded intervals but are not continuous. Differing sample times and finite sample processing delays can be incorporated into the model (for a processing delay $k$, replace $x_{j}(m T)$ by $x_{j}(m T-k)$ in (5.1)).

\section{Conclusions}

A sufficient condition for the stability of the equilibrium points of differential systems with time-varying delays has been derived. The resulting stability tests do not depend strongly upon the forms of the time delay functions; indeed, if these functions are all bounded, the tests are completely independent of them. This is an important feature when determining the stability of models of real systerns for which the time delay parameters are difficult to estimate. 


\section{Acknowledgements}

The author wishes to thank Professor Brian Anderson for suggesting the sampled-data interaction example and for other helpful comments. This work was supported by Rothmans University Endowment.

\section{References}

[1] B. D. O. Anderson, "Time delays in large scale systems" (submitted for publication).

[2] M. H. Chang and P. T. Lin, "Maximum principles for control systems described by measure functional differential equations", J. Opt. Theory and Applic. 15 (1975), 517-531.

[3] P. C. Das and R. A. Sharma, "On optimal controls for measure delay differential equations", SIAM J. Control 9 (1971), 43-61.

[4] J. K. Hale, Functional differential equations (Springer Verlag, New York, 1971).

[5] R. M. Lewis and B. D. O. Anderson, "Insensitivity of a class of non-linear compartmental systems to the introduction of arbitrary time delays", IEEE Circuits and Systems (to appear).

[6] J. Warga, Optimal control of differential and functional equations (Academic Press, New York, 1972).

Department of Electrical Engineering

University of Newcastle

Newcastle

NSW $2308 \dagger$

$\dagger$ Present address: Research and Technology Centre, John Lysaght (Australia), P.O. Box 77, Port Kembla, N.S.W. 2505. 